\title{
Activities and Roles Played by Architects in the Reconstruction of Hiroshima after its Atomic Bombing
}

\author{
Ming $\mathrm{Li}^{1 *}$ and David Richard Neill ${ }^{2}$ \\ 1 JangHo Architecture College, Northeastern University \\ 2 Okayama University of Science \\ *Corresponding Author, Email: liming8@hotmail.com
}

Received: Dec 15, 2016; Accepted: April 10, 2018

Key words: Stricken city, Reconstruction construction, Hiroshima, Urban Design, Architect, Construction documents, Role

Abstract: As seen historically, a War or a Natural Disaster brought devastating destruction to the place where the misfortune took place. The Atomic bombing of Hiroshima and The Great East Japan Earthquake, The Chins Tangshan Earthquake and The Great Wenchuan Earthquake are the cases. The function of the Relief to the cities suffered the disasters and the function of the architects' activities in construction rebuilding should never be overlooked. Hiroshima is about to begin its 70th year after the atomic bombing. The history of the restoration of Hiroshima will be close referenced to the reconstruction of the areas suffering from natural disasters in the future. This paper taking the reconstruction of the Hiroshima as objects of this study, researching by documentary investigations and interviews and investigations on the spot, analyzing reconstruction of the seismic areas and the architects' activities as well as the function of these consequent effects. Through the research following findings were obtained. We can observe that most of the building activity that occurred in the burned ruins immediately after the bombing event was performed by local design offices. The vigorous activity of the local design offices deserves attention and should not be overlooked as a subject of research to better understand the reconstruction of the local city. Although many buildings in the reconstruction process were constructed by others, a major role was played by local design offices. On August 06, 1949, the Hiroshima Peace Memorial City Construction Law was promulgated and two major competitions - the Peace Memorial Cathedral competition and the Hiroshima Peace Center competition-were conducted. These competitions not only played a significant role in promoting the full-scale reconstruction of Hiroshima but also played a major role in the development of post-war apanese architecture. From the early mid-1950s, when the Hiroshima Peace Center and the Peace Memorial Cathedral began to be built, we can observe that a non-Hiroshima Japanese architect or the design office and leading general contractors began to seriously enter the architectural arena in Hiroshima. 


\section{RESEARCH PURPOSE AND SIGNIFICANCE}

\subsection{Background and objective}

As seen historically, it is often in war and natural disasters that cities become ruins in mere seconds, e.g., the city of Hiroshima, destroyed by an atomic bomb in World War II, and the Japanese northeast cities, devastated by the 2011 Tohoku earthquake. With the restoration of these affected cities, the role of an architectural engineer in this reconstruction cannot be ignored. Hiroshima is about to begin its 70th year after the atomic bombing. The reconstruction history of Hiroshima that has risen from the ruins will certainly become a valuable reference for post-earthquake reconstruction in the Tohoku area. Seventy years after exposure to radiation from which no one thought that anything could survive in Hiroshima, the city has been reconstructed under the gaze of the world, becoming an architectural Mecca that beckons visitors from all over Japan. Nevertheless, this popularity is attributed to its historical significance. Hiroshima was the first city to be targeted by the first atomic bomb, which was dropped from the sky on August 06, 1945. The historical significance of the atomic bombings, which have been discussed by other sociological researchers, is well known to many people. Additionally, the reconstruction process has been noted in many newspapers and war-damage reconstruction magazines. For example, multiple studies have focused on the Peace Memorial Park in Hiroshima, designed by Kenzo Tange, and on the Hiroshima World Peace Memorial Cathedral, designed by Togo Murano. However, there has been little mention of the activities of local Hiroshima architects and design offices as well as other architects in Japan. Yoshinari Kouchi, an engineer in the prewar Manchukuo Postal Service Accounting Department of Buildings Department who lived in Hiroshima after the war, played a major role in the reconstruction design of Hiroshima ( $\mathrm{Li}$ and Ishimaru, 2007). The activities of the local architects and design offices cannot be ignored when discussing the reconstruction of Hiroshima.

Focusing on the reconstruction activities of the architects, this study investigates the design offices and construction companies in Hiroshima and attempts to clarify the role of an architectural engineer and the relevancy of construction documents in buildings that rose from the post-war ruins.

\section{$1.2 \quad$ Study method}

First, take Hiroshima as an object and collect information related to postwar reconstruction through a literature survey. The literature survey focuses on the documents related to the post-war reconstruction issued by the state and local government organs. Especially through the investigation of official news reports on the content of the extracted information about the reconstruction of Hiroshima. At the same time, we investigate the publications and papers related to post-war reconstruction by civil circulation and collect information about buildings and architects' activities after the war reconstruction. Second, we analyze the collected data, try to establish a database of the reconstructed building and its associated architects, and make a chart analysis of the activities of the buildings and architects in the first 10 years after the reconstruction. Third, the architectural activities of the architects are classified, mainly by foreign 
architects and local architects. Finally, to clarify the architectural activities and its role of the architects during the reconstruction of Hiroshima.

\subsection{Related study}

There is a lot of research and achievements in the field of sociology and Economics on the issue of war or natural disasters. However, there are few studies in the field of architecture for the rejuvenation of the war disaster or the reconstruction of the earthquake-stricken areas. After the earthquake in northeastern Japan in 2011, the history of earthquake revival began to be emphasized in the field of architecture. The achievements in the history of the war and the history of the reconstruction of the earthquake rejuvenation are more prominent in Japan. The whole city of Japan, the defeated country of the Second World War, was destroyed by destruction. The revival of the war is a major challenge facing Japan, and its revival is one of the most important parts of the history of Japan's post-war architecture. In 1946, the Ministry of construction of Japan construction province carried out a comprehensive investigation on the construction of the disaster recovery in various cities (Japanese Ministry of Construction, 1959). In the survey report, the reconstruction planning and other important cases of all cities in the 15 years after the war were recorded in detail. In addition, each local government records detailed records concerning the Renaissance building deeds. In particular, there are a lot of research results on the construction of the disaster recovery in Hiroshima (Hiroshima Institute of Architects, 1956; Hiroshima Institute of Architects, 1962; Hiroshima Municipal Government, 1985). These books detail the architectural drawings and reconstruction plans of Hiroshima from 1945 to 1962, which are valuable materials for studying and understanding Hiroshima's reconstruction. The theme of the 1997 National Congress of the Japanese Architectural Society was "the revival of Hiroshima", and the history of the reconstruction of the war disaster was taken seriously by the architectural circle. In Japan, the earthquake country, the research on the revival of the earthquake is very extensive and substantial. The great Kanto earthquake (1923), the Great Hanshin earthquake (1995), Tohoku earthquake and tsunami (2011), instantly destroyed the whole district or town, and to human life brought great disaster. Japanese Ministry of Construction (1928) described 5 years after the great Kanto earthquake to reconstruction in Tokyo overview. Tokyo Municipal Government (1932) describes the process of formulating regional planning for the rejuvenation of the city in Tokyo. Tanaka (2006) published research on the revival and living space of the earthquake in Tokyo. Koshizawa and Goto (2011) have published years of research on the reconstruction of Tokyo. Kaji, Izumi, et al. (2012); Yamamoto (2014); Fukushima University International Disaster Revival Research Team (2014); Fujimori (1982), and so on, the research of disaster recovery is very extensive. There are few studies on the architectural activities of the architects during the reconstruction of the disaster areas. The author and Norooki Ishimaru in the last 10 years have researched local architects and made certain achievements (Li and Ishimaru, 2014; Li and Ishimaru, 2007; Li and Ishimaru, 2006; Ishimaru, Li, et al. 2002).

This paper is to clarify the activities and characteristics of the activities of architects or architectural firms in the reconstruction of Hiroshima. Moreover, through these studies, we try to add new content to the research field of urban renewal and construction. 


\section{HIROSHIMA RECONSTRUCTION PROCESS}

Except for a small part, almost entire city center of Hiroshima was process and reconstruction of the city center, the skeleton of the city that was formed at Hiroshima's founding as a castle town was to experience a fundamental remodeling.

The reconstruction of the Hiroshima city center represented an attempt to rise from the ruins. Figure 1 show the completed building. Herein, we focused on the reconstruction process by performing a literature review (Hiroshima Municipal Government, 1985; Ishimaru, 1990). The first major action is taken to rebuild the city after the war was the development of the war-damaged reconstruction plan and its project implementation. In the Hiroshima City Planning Division, a reconstruction plan was developed by the manager Teizo Takeshige immediately after the war. A reconstruction office was established in this city in January 1946. It worked on the reconstruction plan under the supervision of Satoshi Nagashima, the reconstruction director. Nagashima, associated with the city reconstruction office, became the director general with the responsibility for organizing the Hiroshima reconstruction council.

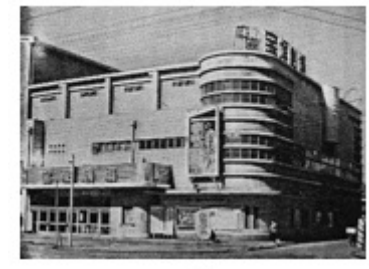

(a)

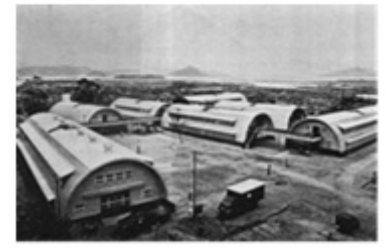

(d)

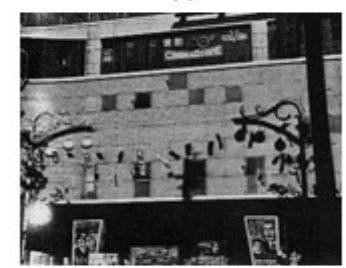

(g)

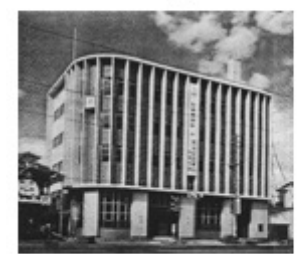

(j)

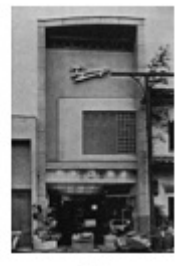

(b)

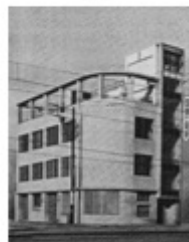

(e)

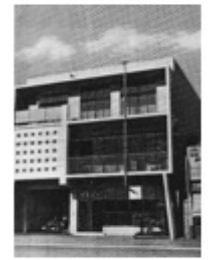

(h)

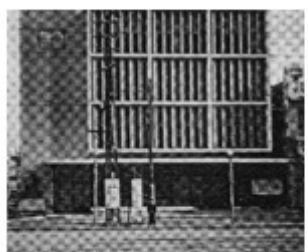

(k)

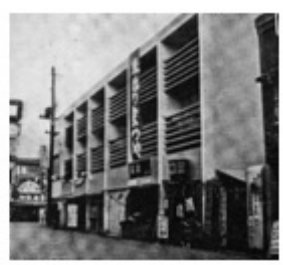

(c)

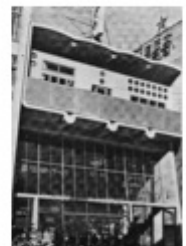

(f)

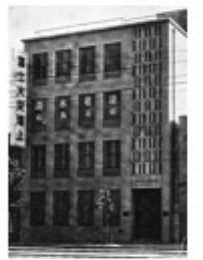

(i)

Figure 1. Reconstruction of Hiroshima City. (a) Hiroshima Takarazuka (1951 completion, Fujita-gumi of design). (b) Tajimaya (1952 completion Asano-gumis of design). (c) Mutsumi shop mortgage (1953 clay architect office, the design of the Hiroshima Prefectural Housing Corporation). (d) ABCC (1953 completion, ABCC Construction Department of the design).

(e) Hiroshima Central Building (1953 completion, Ryotaro Tanaka of design). (f) Shimamura Motors (1953 completion, Isamu Ueno design). (g) Oriental masterpiece theater (1954 completion, Isamu Ueno design). (h) Nakamura Kosha (1954 completion, Isamu Ueno 
design). (i) Fuji Fire and Marine Insurance Hiroshima branch (1954 completion, the design of the Shimizu Corporation). (j) Hiroshima Building (1954 completion, Nikken Sekkei of design). (k) Hiroshima Credit Bank Dobashi branch (1955, Kimura, Toshio of design)

A process to discuss the reconstruction plan was established on February 25 of the same year. During this period and after, many initiatives and proposals associated with the reconstruction plan of Hiroshima were submitted and lively discussion abounded. For example, more than 30 cases for urban relocation on the ground-breaking concept of "Hiroshima 1965" can be counted. After these spectacular concepts had been advanced, the plan of the war-damaged reconstruction institute and Hiroshima prefecture for Hiroshima city was adjusted. Finally, after a considerable amount of discussion on city planning by the Hiroshima Regional Board, Hiroshima reconstruction urban planning was established.

The reconstruction project was scheduled to be completed under the first five-year plan; however, a serious issue reduced the pace of the progress: after the bombing event, Hiroshima suffered financial crisis.

Considering this background, the national government played a decisive role in the reconstruction of the Hiroshima city. On August 06, 1949, it promulgated the Hiroshima Peace Memorial City Construction Law (Law No. 219), hereinafter referred to as "peace urban law." With the enactment of the peace urban law, the reconstruction urban planning completed up to that time was discarded. The reconstruction project thus changed and became known as the "Hiroshima Peace Memorial City Construction Plan." It was adopted in March 1952. In this way, substantial assistance from the national government came to support the Peace Memorial City Construction of Hiroshima.

Hiroshima become the subject of world attention. It received donations from all over the world. On the domestic side, investment in the construction of public buildings and construction companies increased. In 1946, the postwar local Akatsuki Design Office built the Asahi Newspaper Hiroshima Bureau building and Hiroshima Priest Hall. In 1947, the Hiroshima Woman's Gakuen Junior High School and High School and the Koryo Junior High School were completed. In 1948, the Sanyo Junior High School, the Hiroshima Woman's School auditorium, the Mainichi Shimbun Hiroshima Bureau, and the Children's Cultural Hall were completed. In 1949, the peace urban law was applied to support architectural design competitions, including that for the Peace Memorial Park. In the competition, the plan of Kenzo Tange's group was awarded the first prize. In the Peace Memorial City Construction Plan, Nakajima Park was to be developed as a memorial facility. The construction of the Atomic Bomb Museum and the Peace Memorial Hall was promoted since 1950. During 1951, in Takarazuka Hiroshima (Figure 1 (a)), the Hiroshima General Grand Main Building, the Agricultural Cooperative building, the Hiroshima International Trade and Industry Bureau, the Hiroshima Family Court, the Mizuno assembly building, the Hiroshima City Central Wholesale Market, and other structures, such as the Peace Bridge, were built one after another. During 1952 (Figure 1 (b)), in Tajimaya, the Hiroshima Department Store, Kokutai-ji High School, the Nippon Fire \& Marine Insurance Co., Ltd. Building, and the Hiroshima Bank Motokawa Branch were completed. In 1953, the Hiroshima City Children's Library, housing with Mutsumi Stores (Figure 1 (c)), the Atomic Bomb Casualty Commission (Figure 1 (d)), the Hiroshima Central Building (Figure 1 (e)), Shimamura Motors (Figure 1 
(f)), and the American Dormitory were completed. In 1954, the East Masterpiece Theater (Figure 1 (g)), Nakamura Kosha (Figure 1 (h)), the Fuji Fire and Marine Insurance Hiroshima branch (Figure 1 (i)), and the Hiroshima Building (Figure 1 (j)) were built. In 1955, the Hiroshima Credit Bank Dobashi branch (Figure $1(\mathrm{k})$ ) and the Hiroshima Women's Junior College were completed. In 1956, the prefectural office building and the construction of the Tenmaya Department Store were completed. By around 1957, the Hiroshima Municipal Stadium was built in Moto machi, the socalled center of the city.

The Hiroshima prefectural government and the bus center had already been built in Moto machi, and the old military land was used for a new city center formation and civic entertainment facilities. Numerous constructions, ranging from schools and bank buildings to housing, occurred at this time. In this way, the center of Hiroshima was substantially reconstructed.

\section{RECONSTRUCTION AND ARCHITECTURAL ENGINEERS}

Which types of buildings were constructed in Hiroshima immediately after the bombing? A previous study $(\mathrm{Li}, 2012)$ summarized the post-war decade of the working architects or design offices and their work, and we supplement that presentation here with the information derived from a new literature survey and interviews (Hiroshima Institute of Architects, 1956; Hiroshima Institute of Architects, 1962; Hiroshima Institute of Architects, 1983; Hiroshima Municipal Government, 1985) as summarized in Table 1.

Table 1. Reconstruction architecture and architects (1945-1955)

\begin{tabular}{|c|c|c|c|c|}
\hline No. & $\begin{array}{l}\text { Year of } \\
\text { construction }\end{array}$ & Building name & $\begin{array}{l}\text { Architect or design } \\
\text { office }\end{array}$ & Remarks \\
\hline 1 & 1946 & Priest's Hall & Akatsuki Design Office & $\bullet$ \\
\hline 2 & 1946 & $\begin{array}{l}\text { Asahi Broadcasting } \\
\text { Corporation } \\
\text { Hiroshima bureau }\end{array}$ & Akatsuki Design Office & $\bullet$ \\
\hline 3 & 1947 & $\begin{array}{l}\text { Governor of } \\
\text { Hiroshima } \\
\text { Prefecture official } \\
\text { residence }\end{array}$ & $\begin{array}{l}\text { Hiroshima Prefecture } \\
\text { Buildings Department } \\
\text { Division }\end{array}$ & $\bullet$ \\
\hline 4 & 1947 & $\begin{array}{l}\text { Hiroshima Jogakuin } \\
\text { High School }\end{array}$ & Akatsuki Design Office & $\bullet$ \\
\hline 5 & 1947 & Koryo High School & Akatsuki Design Office & $\bullet$ \\
\hline 6 & 1948 & $\begin{array}{l}\text { Sanyo Junior High } \\
\text { School }\end{array}$ & Akatsuki Design Office & $\bullet$ \\
\hline 7 & 1948 & $\begin{array}{l}\text { Hiroshima Jogakuin } \\
\text { Auditorium }\end{array}$ & Akatsuki Design Office & $\bullet$ \\
\hline 8 & 1948 & $\begin{array}{l}\text { Mainichi Shimbun } \\
\text { Hiroshima Bureau }\end{array}$ & Akatsuki Design Office & $\bullet$ \\
\hline 9 & 1948 & $\begin{array}{l}\text { Hiroshima } \\
\text { Children's Cultural } \\
\text { Hall }\end{array}$ & Akatsuki Design Office & $\bullet$ \\
\hline 10 & 1948 & $\begin{array}{l}\text { Religious high } \\
\text { school new school } \\
\text { building }\end{array}$ & $\begin{array}{l}\text { Saburo Sugita Design } \\
\text { Office }\end{array}$ & $\bullet$ \\
\hline 11 & 1949 & $\begin{array}{l}\text { Hiroshima Gas } \\
\text { Building }\end{array}$ & Akatsuki Design Office & $\bullet$ \\
\hline 12 & 1949 & $\begin{array}{l}\text { Religious high } \\
\text { school new school } \\
\text { building }\end{array}$ & $\begin{array}{l}\text { Saburo Sugita Design } \\
\text { Office }\end{array}$ & $\bullet$ \\
\hline
\end{tabular}




\begin{tabular}{|c|c|c|c|c|}
\hline & $\begin{array}{l}\text { Year of } \\
\text { construction }\end{array}$ & Building name & $\begin{array}{l}\text { Architect or design } \\
\text { office }\end{array}$ & Remarks \\
\hline 13 & 1949 & $\begin{array}{l}\text { Religious high } \\
\text { school special } \\
\text { classroom new } \\
\text { construction }\end{array}$ & $\begin{array}{l}\text { Saburo Sugita Design } \\
\text { Office }\end{array}$ & $\bullet$ \\
\hline 14 & 1949 & $\begin{array}{l}\text { Religious high } \\
\text { school auditorium }\end{array}$ & $\begin{array}{l}\text { Saburo Sugita Design } \\
\text { Office }\end{array}$ & $\bullet$ \\
\hline 15 & 1949 & $\begin{array}{l}\text { Funakoshi Junior } \\
\text { High School } \\
\text { building }\end{array}$ & $\begin{array}{l}\text { Saburo Sugita Design } \\
\text { Office }\end{array}$ & $\bullet$ \\
\hline 16 & 1950 & $\begin{array}{l}\text { Taisho Marine Fire } \\
\text { Insurance Hiroshima } \\
\text { Branch }\end{array}$ & Akatsuki Design Office & $\bullet$ \\
\hline 17 & 1950 & $\begin{array}{l}\text { Electrical } \\
\text { Association } \\
\text { Hiroshima Branch } \\
\text { Office building }\end{array}$ & $\begin{array}{l}\text { Saburo Sugita Design } \\
\text { Office }\end{array}$ & $\bullet$ \\
\hline 18 & 1950 & $\begin{array}{l}\text { Hirowata } \\
\text { Headquarters }\end{array}$ & $\begin{array}{l}\text { Saburo Sugita Design } \\
\text { Office }\end{array}$ & $\bullet$ \\
\hline 19 & 1950 & $\begin{array}{l}\text { Minami-cho, civil } \\
\text { servants quarters }\end{array}$ & $\begin{array}{l}\text { Chugoku Shikoku } \\
\text { Regional Construction } \\
\text { Bureau Buildings } \\
\text { Department Division }\end{array}$ & $\bullet$ \\
\hline 20 & 1950 & $\begin{array}{l}\text { Hiroshima Minami } \\
\text { High School } \\
\text { extension }\end{array}$ & Shirahani Design Office & $\bullet$ \\
\hline 21 & 1950 & $\begin{array}{l}\text { Hiroshima Danbara } \\
\text { Junior High School } \\
\text { extension }\end{array}$ & Shirahani Design Office & $\bullet$ \\
\hline 22 & 1950 & $\begin{array}{l}\text { Amagi Inn new } \\
\text { construction }\end{array}$ & Shirahani Design Office & $\bullet$ \\
\hline 23 & 1950 & $\begin{array}{l}\text { Cabaret Petra new } \\
\text { construction }\end{array}$ & Shirahani Design Office & $\bullet$ \\
\hline 24 & 1951 & $\begin{array}{l}\text { Hiroshima } \\
\text { Comprehensive } \\
\text { Grand main building }\end{array}$ & $\begin{array}{l}\text { Hiroshima Prefecture } \\
\text { Buildings Department } \\
\text { Division }\end{array}$ & $\bullet$ \\
\hline 25 & 1951 & $\begin{array}{l}\text { Agricultural } \\
\text { Cooperative } \\
\text { Building }\end{array}$ & Akatsuki Design Office & $\bullet$ \\
\hline 26 & 1951 & $\begin{array}{l}\text { Hiroshima Bureau of } \\
\text { International Trade } \\
\text { and Industry }\end{array}$ & $\begin{array}{l}\text { Chugoku Shikoku } \\
\text { Regional Construction } \\
\text { Bureau Buildings } \\
\text { Department Division }\end{array}$ & $\bullet$ \\
\hline 27 & 1951 & $\begin{array}{l}\text { Hiroshima Family } \\
\text { Court }\end{array}$ & Supreme Court & $\circ$ \\
\hline 28 & 1951 & $\begin{array}{l}\text { Mizuno Assembly } \\
\text { Building }\end{array}$ & Mizuno-gumi & $\circ$ \\
\hline 29 & 1951 & $\begin{array}{l}\text { Hiroshima Central } \\
\text { Wholesale Market }\end{array}$ & $\begin{array}{l}\text { Hiroshima City } \\
\text { Buildings Department } \\
\text { Division }\end{array}$ & $\bullet$ \\
\hline 30 & 1951 & $\begin{array}{l}\text { Hiroshima } \\
\text { Takarazuka }\end{array}$ & Fujita-gumi & $\circ$ \\
\hline 31 & 1951 & $\begin{array}{l}\text { Mitsubishi Bank } \\
\text { Hiroshima Branch }\end{array}$ & $\begin{array}{l}\text { Mitsubishi Bank } \\
\text { Maintenance } \\
\text { Department \& Fujita- } \\
\text { gumi }\end{array}$ & $\circ$ \\
\hline 32 & 1951 & $\begin{array}{l}\text { Sanyo High School } \\
\text { special classroom }\end{array}$ & $\begin{array}{l}\text { Saburo Sugita Design } \\
\text { Office }\end{array}$ & $\bullet$ \\
\hline 33 & 1951 & $\begin{array}{l}\text { Sanyo High School } \\
\text { auditorium }\end{array}$ & $\begin{array}{l}\text { Saburo Sugita Design } \\
\text { Office }\end{array}$ & $\bullet$ \\
\hline 34 & 1951 & $\begin{array}{l}\text { Eba Junior High } \\
\text { School building }\end{array}$ & $\begin{array}{l}\text { Saburo Sugita Design } \\
\text { Office }\end{array}$ & $\bullet$ \\
\hline
\end{tabular}




\begin{tabular}{ll}
\hline No. & $\begin{array}{l}\text { Year of } \\
\text { construction }\end{array}$ \\
35 & 1951 \\
36 & 1951 \\
37 & 1951
\end{tabular}

381951

391951

$40 \quad 1951$

$41 \quad 1951$

421952

431952

$44 \quad 1952$

451952

$46 \quad 1952$

$47 \quad 1952$

$48 \quad 1952$

491952

501952

$51 \quad 1952$

521952

531952

541952

551952

561952

571953

581953

$\begin{array}{lll}\text { Building name } & \begin{array}{l}\text { Architect or design } \\ \text { office }\end{array} & \text { Remarks } \\ \text { Futaba Junior High } & \begin{array}{l}\text { Saburo Sugita Design } \\ \text { School building }\end{array} & \bullet \\ \text { Office } & \\ \text { Peace Bridge } & \text { Isamu Noguchi } & \circ\end{array}$

Hiroshima Nakajima Shirahani Design Office •

Elementary School

extension

The second mall new Shirahani Design Office construction before

Hiroshima Station

Hiroshima Shirahani Design Office

Yokogawa-cho, mall

new construction

Matsuda House new Shirahani Design Office

construction

Takeya-cho Shirahani Design Office

Lutheran Church

new construction

Cenotaph for A- Kenzo Tange o

bomb victims

Kokutai-ji High Hiroshima Prefecture

School

Buildings Department

Division

Hiroshima Akatsuki Design Office

Department Store

Hiroshima Higashi Hiroshima City

Public Health Center Buildings Department

Division

Fujita building Fujita-gumi

Fuji Bank Hiroshima Fuji Bank Maintenance o

Branch

Department\&Fujita -

gumi

Hiroshima City Hiroshima City

Waterworks Bureau Waterworks Facilities

Division

Hiroshima Railway Railway Administration $\circ$

Administration

Facilities Department

Building Division

JNR Futabanosato Railway Administration o Facilities Department

Building Division

Tajimaya Asano-gumi

Nippon Fire \& Toda-gumi

Marine Insurance

Co., Ltd. Building

Hiroshima Bank

Honkawa Branch

Futaba Junior High $\quad$ Saburo Sugita Design

School special Office

classroom new

construction

Hiroshima Shirahani Design Office

Prefectural Housing

Corporation

Mutsumi house

construction

Xintenti Theater new Shirahani Design Office $\bullet$

construction

Hiroshima City

Kenzo Tange

Children's Library

Yashima Car Shop Saburo Sugita Design

Office 


\begin{tabular}{|c|c|c|c|c|}
\hline & $\begin{array}{l}\text { Year of } \\
\text { construction }\end{array}$ & Building name & $\begin{array}{l}\text { Architect or design } \\
\text { office }\end{array}$ & $\overline{\text { Remarks }}$ \\
\hline 59 & 1953 & $\begin{array}{l}\text { Funakoshi } \\
\text { Elementary School } \\
\text { auditorium }\end{array}$ & $\begin{array}{l}\text { Saburo Sugita Design } \\
\text { Office }\end{array}$ & $\bullet$ \\
\hline 60 & 1953 & $\begin{array}{l}\text { Funakoshi } \\
\text { Elementary School } \\
\text { building }\end{array}$ & $\begin{array}{l}\text { Saburo Sugita Design } \\
\text { Office }\end{array}$ & $\bullet$ \\
\hline 61 & 1953 & $\begin{array}{l}\text { ABCC American } \\
\text { dormitory }\end{array}$ & Kunio Maekawa & $\circ$ \\
\hline 62 & 1953 & Shimamura Motors & Isamu Ueno & $\bullet$ \\
\hline 63 & 1953 & $\begin{array}{l}\text { Food Industrial } \\
\text { Research Institute }\end{array}$ & $\begin{array}{l}\text { Hiroshima Prefecture } \\
\text { Buildings Department } \\
\text { Division }\end{array}$ & $\bullet$ \\
\hline 64 & 1953 & $\begin{array}{l}\text { Chiken and } \\
\text { Materials Proving } \\
\text { Ground }\end{array}$ & $\begin{array}{l}\text { Chugoku Shikoku } \\
\text { Regional Construction } \\
\text { Bureau Buildings } \\
\text { Department Division }\end{array}$ & $\bullet$ \\
\hline 65 & 1953 & $\begin{array}{l}\text { Hiroshima Minami } \\
\text { Telephone Station }\end{array}$ & $\begin{array}{l}\text { Japan Telephone Public } \\
\text { Corporation Facilities } \\
\text { Department }\end{array}$ & $\circ$ \\
\hline 66 & 1953 & $\begin{array}{l}\text { Hiroshima Central } \\
\text { Building }\end{array}$ & Ryotaro Tanaka & $\circ$ \\
\hline 67 & 1953 & $\begin{array}{l}\text { Atomic Bomb } \\
\text { Casualty } \\
\text { Commission }\end{array}$ & $\begin{array}{l}\text { ABCC Construction } \\
\text { Department }\end{array}$ & $\circ$ \\
\hline 68 & 1953 & $\begin{array}{l}\text { Chugoku Bank } \\
\text { Hiroshima Branch }\end{array}$ & Takenaka Corporation & $\circ$ \\
\hline 69 & 1953 & $\begin{array}{l}\text { Daiwa Bank } \\
\text { Hiroshima Branch }\end{array}$ & Takenaka Corporation & $\circ$ \\
\hline 70 & 1953 & $\begin{array}{l}\text { Chugoku Telecom } \\
\text { Phone Hall }\end{array}$ & $\begin{array}{l}\text { Ghugoku } \\
\text { Telecommunications } \\
\text { Stations Building } \\
\text { Department }\end{array}$ & $\bullet$ \\
\hline 71 & 1953 & $\begin{array}{l}\text { Hiroshima Teishin } \\
\text { Hospital length } \\
\text { dormitory }\end{array}$ & $\begin{array}{l}\text { Hiroshima Postal } \\
\text { Service Building } \\
\text { Department }\end{array}$ & $\bullet$ \\
\hline 72 & 1953 & $\begin{array}{l}\text { Hiroshima } \\
\text { Prefectural Housing } \\
\text { Corporation Fukuma } \\
\text { housing }\end{array}$ & Shirahani Design Office & $\bullet$ \\
\hline 73 & 1953 & $\begin{array}{l}\text { Hiroshima } \\
\text { Prefectural Housing } \\
\text { Corporation Hisada } \\
\text { housing }\end{array}$ & Shirahani Design Office & $\bullet$ \\
\hline 74 & 1953 & $\begin{array}{l}\text { Hiroshima } \\
\text { Prefectural Housing } \\
\text { Corporation } \\
\text { Kotobuki housing }\end{array}$ & Shirahani Design Office & $\bullet$ \\
\hline 75 & 1953 & $\begin{array}{l}\text { Hiroshima } \\
\text { Prefectural Housing } \\
\text { Corporation Ohno } \\
\text { housing }\end{array}$ & Shirahani Design Office & $\bullet$ \\
\hline 76 & 1953 & $\begin{array}{l}\text { Hiroshima City } \\
\text { Auditorium new } \\
\text { construction }\end{array}$ & Shirahani Design Office & $\bullet$ \\
\hline 77 & 1953 & $\begin{array}{l}\text { Hiroshima } \\
\text { Prefectural Housing } \\
\text { Corporation largely } \\
\text { residential }\end{array}$ & Shirahani Design Office & $\bullet$ \\
\hline 78 & 1954 & $\begin{array}{l}\text { World Peace } \\
\text { Memorial Cathedral }\end{array}$ & $\begin{array}{l}\text { Murano \& Mori Design } \\
\text { Office }\end{array}$ & $\circ$ \\
\hline 79 & 1954 & Honkawa & Saburo Sugita Design & $\bullet$ \\
\hline
\end{tabular}




\begin{tabular}{|c|c|c|c|c|}
\hline \multirow{2}{*}{\multicolumn{2}{|c|}{$\begin{array}{l}\text { No. Year of } \\
\text { construction }\end{array}$}} & Building name & $\begin{array}{l}\text { Architect or design } \\
\text { office }\end{array}$ & $\overline{\text { Remarks }}$ \\
\hline & & $\begin{array}{l}\text { Elementary School } \\
\text { building }\end{array}$ & Office & \\
\hline 80 & 1954 & $\begin{array}{l}\text { Yasudagakuen } \\
\text { Library }\end{array}$ & $\begin{array}{l}\text { Saburo Sugita Design } \\
\text { Office }\end{array}$ & $\bullet$ \\
\hline 81 & 1954 & Nakamura Kosha & Isamu Ueno & $\bullet$ \\
\hline 82 & 1954 & $\begin{array}{l}\text { Oriental Masterpiece } \\
\text { Theater }\end{array}$ & Isamu Ueno & $\bullet$ \\
\hline 83 & 1954 & Skating Arena & Isamu Ueno & $\bullet$ \\
\hline 84 & 1954 & $\begin{array}{l}\text { Hiroshima City } \\
\text { Fuchu-cho } \\
\text { government office }\end{array}$ & $\begin{array}{l}\text { Hiroshima Prefecture } \\
\text { Buildings Department } \\
\text { Division }\end{array}$ & $\bullet$ \\
\hline 85 & 1954 & $\begin{array}{l}\text { Social Insurance } \\
\text { Hiroshima City } \\
\text { Hospital }\end{array}$ & Akatsuki Design Office & $\bullet$ \\
\hline 86 & 1954 & $\begin{array}{l}\text { Chugoku Electrical } \\
\text { Work }\end{array}$ & Fujita-gumi & ० \\
\hline 87 & 1954 & $\begin{array}{l}\text { Taisei Hiroshima } \\
\text { Employees } \\
\text { Apartment }\end{array}$ & $\begin{array}{l}\text { Taisei Corporation } \\
\text { Hiroshima Branch }\end{array}$ & $\square$ \\
\hline 88 & 1954 & Kyobashi Hall & $\begin{array}{l}\text { Taisei Corporation } \\
\text { Hiroshima Branch }\end{array}$ & $\square$ \\
\hline 89 & 1954 & Koi housing & $\begin{array}{l}\text { Taisei Corporation } \\
\text { Hiroshima Branch }\end{array}$ & $\square$ \\
\hline 90 & 1954 & $\begin{array}{l}\text { Hiroshima Post } \\
\text { Office Noborimachi } \\
\text { Branch }\end{array}$ & $\begin{array}{l}\text { Hiroshima Postal } \\
\text { Service Building } \\
\text { Department }\end{array}$ & $\bullet$ \\
\hline 91 & 1954 & $\begin{array}{l}\text { Fuji Fire and Marine } \\
\text { Insurance Hiroshima } \\
\text { Branch }\end{array}$ & Shimizu Corporation & ○ \\
\hline 92 & 1954 & $\begin{array}{l}\text { Hiroshima Every } \\
\text { Day Hall }\end{array}$ & $\begin{array}{l}\text { Yoshinari Kouchi } \\
\text { Design Office }\end{array}$ & $\bullet$ \\
\hline 93 & 1954 & Mr. I House & $\begin{array}{l}\text { Yoshinari Kouchi } \\
\text { Design Office }\end{array}$ & $\bullet$ \\
\hline 94 & 1954 & Hiroshima Building & Nikken Design & ○ \\
\hline 95 & 1954 & Monopoly Cafeteria & Nikken Design & $\circ$ \\
\hline 96 & 1954 & ShibaSo building & Obayashi-gumi & $\circ$ \\
\hline 97 & 1954 & $\begin{array}{l}\text { Office Hiroshima } \\
\text { University Faculty of } \\
\text { Letters }\end{array}$ & $\begin{array}{l}\text { Ministry Of Education } \\
\text { Educational Facilities } \\
\text { Department Hiroshima } \\
\text { Construction Office }\end{array}$ & $\square$ \\
\hline 98 & 1954 & Suzuki shop & Onoki Construction & $\bullet$ \\
\hline & 1954 & $\begin{array}{l}\text { First Bank } \\
\text { Hiroshima Branch }\end{array}$ & $\begin{array}{l}\text { Koji Nishimura Design } \\
\text { Office }\end{array}$ & $\circ$ \\
\hline 100 & 1954 & $\begin{array}{l}\text { Industrial Bank of } \\
\text { Japan Hiroshima } \\
\text { Branch }\end{array}$ & $\begin{array}{l}\text { Toshiro Yamashita } \\
\text { Design Office }\end{array}$ & ○ \\
\hline 101 & 1954 & $\begin{array}{l}\text { Hiroshima } \\
\text { Municipal Asano } \\
\text { Library }\end{array}$ & Kikuji Ishimoto & $\circ$ \\
\hline 102 & 1954 & Hijiyama shrine & Masao Sasaguchi & ० \\
\hline 103 & 1954 & $\begin{array}{l}\text { Lutheran } \\
\text { Kindergarten }\end{array}$ & ARB Design Office & $\bullet$ \\
\hline 104 & 1954 & $\begin{array}{l}\text { Hiroshima } \\
\text { Municipal Senda- } \\
\text { cho Elementary } \\
\text { School auditorium } \\
\text { new construction }\end{array}$ & Shirahani Design Office & $\bullet$ \\
\hline 105 & 1955 & Peace Memorial & Kenzo Tange & ० \\
\hline 106 & 1955 & $\begin{array}{l}\text { Peace Memorial } \\
\text { Museum }\end{array}$ & Kenzo Tange & ० \\
\hline 107 & 1955 & Hiroshima Women's & Hiroshima Prefecture & $\bullet$ \\
\hline
\end{tabular}




\begin{tabular}{|c|c|c|c|}
\hline $\begin{array}{l}\text { No. Year of } \\
\text { construction }\end{array}$ & $\begin{array}{l}\text { Building name } \\
\text { Junior College }\end{array}$ & $\begin{array}{l}\text { Architect or design } \\
\text { office } \\
\text { Buildings Department } \\
\text { Division }\end{array}$ & Remarks \\
\hline 1081955 & $\begin{array}{l}\text { Fukuromachi } \\
\text { Elementary School }\end{array}$ & $\begin{array}{l}\text { Hiroshima City } \\
\text { Buildings Department } \\
\text { Division }\end{array}$ & $\bullet$ \\
\hline 1091955 & Public toilet & $\begin{array}{l}\text { Hiroshima City } \\
\text { Buildings Department } \\
\text { Division }\end{array}$ & $\bullet$ \\
\hline 1101955 & Religious school & Fujita-gumi & ० \\
\hline 1111955 & $\begin{array}{l}\text { Industrial Bank of } \\
\text { Japan, the Hiroshima } \\
\text { branch company } \\
\text { housing }\end{array}$ & $\begin{array}{l}\text { Saburo Sugita Design } \\
\text { Office }\end{array}$ & $\bullet$ \\
\hline 1121955 & Yokogawa housing & $\begin{array}{l}\text { Taisei Corporation } \\
\text { Hiroshima Branch }\end{array}$ & $\square$ \\
\hline 1131955 & $\begin{array}{l}\text { Dentsu Ujina staff } \\
\text { quarters }\end{array}$ & $\begin{array}{l}\text { Tyuoku } \\
\text { Telecommunications } \\
\text { Stations Building } \\
\text { Department }\end{array}$ & $\bullet$ \\
\hline 1141955 & $\begin{array}{l}\text { Hiroshima Control } \\
\text { Wireless } \\
\text { Interruption office }\end{array}$ & $\begin{array}{l}\text { Tyuoku } \\
\text { Telecommunications } \\
\text { Stations Building } \\
\text { Department }\end{array}$ & $\bullet$ \\
\hline 1151955 & $\begin{array}{l}\text { A-bomb victims } \\
\text { memorial }\end{array}$ & $\begin{array}{l}\text { Tyuoku } \\
\text { Telecommunications } \\
\text { Stations Building } \\
\text { Department }\end{array}$ & $\bullet$ \\
\hline 1161955 & Hiroshima Post Hall & $\begin{array}{l}\text { Hiroshima Postal } \\
\text { Service Building } \\
\text { Department }\end{array}$ & $\bullet$ \\
\hline 1171955 & $\begin{array}{l}\text { Hiroshima Postal } \\
\text { Service Kougo } \\
\text { quarters }\end{array}$ & $\begin{array}{l}\text { Hiroshima Postal } \\
\text { Service Building } \\
\text { Department }\end{array}$ & $\bullet$ \\
\hline 1181955 & $\begin{array}{l}\text { Hiroshima } \\
\text { Notorudamu } \\
\text { Seishingakuen }\end{array}$ & $\begin{array}{l}\text { Shimizu Corporation } \\
\text { Hiroshima Branch }\end{array}$ & $\square$ \\
\hline 1191955 & $\begin{array}{l}\text { Sanwa Bank } \\
\text { Hiroshima Station } \\
\text { before Branch }\end{array}$ & $\begin{array}{l}\text { Shimizu Corporation } \\
\text { Hiroshima Branch }\end{array}$ & $\square$ \\
\hline 1201955 & $\begin{array}{l}\text { Fuchu-cho Central } \\
\text { Community Center }\end{array}$ & $\begin{array}{l}\text { Yoshinari Kouchi } \\
\text { Design Office }\end{array}$ & $\bullet$ \\
\hline 1211955 & Mr. K House & $\begin{array}{l}\text { Yoshinari Kouchi } \\
\text { Design Office }\end{array}$ & $\bullet$ \\
\hline 1221955 & Shanglin & $\begin{array}{l}\text { Yoshinari Kouchi } \\
\text { Design Office }\end{array}$ & $\bullet$ \\
\hline 1231955 & Umetsubo & $\begin{array}{l}\text { Yoshinari Kouchi } \\
\text { Design Office }\end{array}$ & $\bullet$ \\
\hline 1241955 & $\begin{array}{l}\text { Recuperate Institute } \\
\text { (psychiatric } \\
\text { hospitals) }\end{array}$ & $\begin{array}{l}\text { Yoshinari Kouchi } \\
\text { Design Office }\end{array}$ & $\bullet$ \\
\hline 1251955 & $\begin{array}{l}\text { Hiroshima } \\
\text { Prefecture Welfare } \\
\text { credit union }\end{array}$ & ARB Design Office & $\bullet$ \\
\hline 1261955 & $\begin{array}{l}\text { Mazda Motors used } \\
\text { car body repair shop }\end{array}$ & Shirahani Design Office & $\bullet$ \\
\hline 1271955 & $\begin{array}{l}\text { Hiroshima Mazda } \\
\text { Motors office and } \\
\text { factory }\end{array}$ & Shirahani Design Office & $\bullet$ \\
\hline 1281955 & $\begin{array}{l}\text { Peace Memorial } \\
\text { Auditorium }\end{array}$ & Shirahani Design Office & $\bullet$ \\
\hline 1291955 & Shinhiroshima & Shirahani Design Office & $\bullet$ \\
\hline
\end{tabular}




\begin{tabular}{|c|c|c|c|}
\hline $\begin{array}{l}\text { No. Year of } \\
\text { construction }\end{array}$ & Building name & $\begin{array}{l}\text { Architect or design } \\
\text { office }\end{array}$ & Remarks \\
\hline & Hotels & & \\
\hline 1301955 & Sanyo building & Kyoritsu-gumi & ० \\
\hline 1311955 & Chugoku Newspaper & $\begin{array}{l}\text { Murata \& Ohhata } \\
\text { Design Office }\end{array}$ & $\bullet$ \\
\hline 1321955 & Nakazawa Hospital & $\begin{array}{l}\text { Murata \& Ohhata } \\
\text { Design Office }\end{array}$ & $\bullet$ \\
\hline 1331955 & $\begin{array}{l}\text { Chugoku Employees } \\
\text { Apartment }\end{array}$ & $\begin{array}{l}\text { Murata \& Ohhata } \\
\text { Design Office }\end{array}$ & $\bullet$ \\
\hline 1341955 & $\begin{array}{l}\text { Hiroshima } \\
\text { Prefecture Shinren } \\
\text { Kaita City Branch }\end{array}$ & $\begin{array}{l}\text { Murata \& Ohhata } \\
\text { Design Office }\end{array}$ & $\bullet$ \\
\hline
\end{tabular}

Note: Table 1 includes some wooden buildings (rare exceptions) and reinforced concrete buildings, as identified by the main search. In addition, the symbol " $\circ$ " represents a foreign architect and construction organization, "•" represents a local architect and construction organization, and " $\square$ " indicates a branch that was established in Hiroshima, e.g., that of a major general contractor.

As summarized in Table 1, 134 buildings constructed in the decade immediately following the end of World War II along with their designers can be confirmed. Among them, 102 structures were designed by Hiroshima's local architects, architectural offices, and prefectural city maintenance companies. In 32 cases, the buildings were designed by architects neither in Hiroshima itself nor attached to one of the design offices of, for example, the Hiroshima branch office of one of the major general contractors. Based on the details summarized in Table 1 and the reconstruction architecture of the post-war decade, the affiliation of the designers is shown in Figure 2. Affiliations are divided into three categories: local architects or design offices, non-Hiroshima Japanese architects or design offices, and government office maintenance organization. Two periods are considered: 1945-1950 and 1951-1955. As shown in Figure 2, the number of buildings constructed from 1945 to 1950 by non-Hiroshima Japanese architects is 0 , that for the buildings constructed by local Hiroshima architects or design offices is 21 , and that for the buildings constructed by the Government Buildings Department organization is 2. Based on the construction activities from 1951 to 1955, the number of buildings constructed by non-Hiroshima Japanese architects is 32, that for the buildings constructed by local architects or design offices is 61 , and that for the buildings constructed by the government office maintenance organization is 18 .

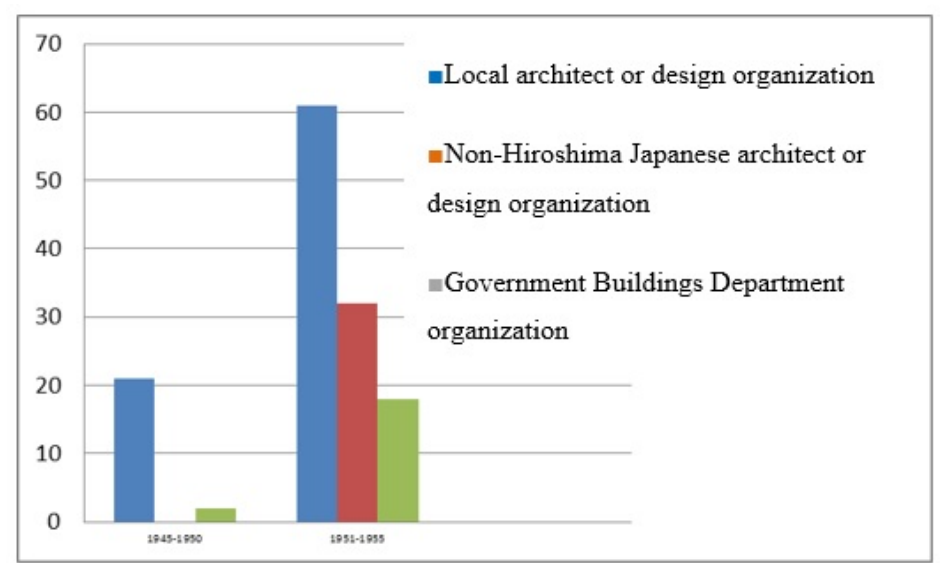

Figure 2. Buildings constructed in the post-war decade by years and by architect or design office 


\section{ACTUAL ACTIVITY OF THE ARCHITECTS AND DESIGN OFFICES}

First, we classify the activities of architects as those performed by local architects, non-Hiroshima Japanese architects, and architects of the government office maintenance organization and show their respective design work in percentage.

A summary of the architectural activity in the post-war decade from 1945 to 1955 is shown in Figure 3. As shown in this figure, local architects were responsible for $61 \%$ of the total construction activity, non-Hiroshima Japanese architects were responsible for $24 \%$, and the government office maintenance organization was responsible for $15 \%$.

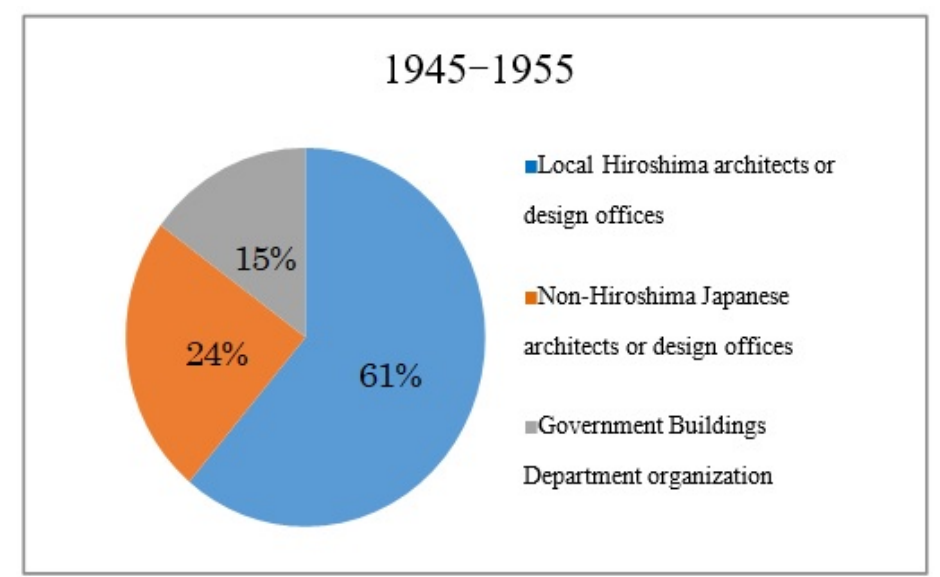

Figure 3. Activity of the architects or design offices in the decade after the war

We further analyze this activity in Figures 4 and Figures 5. A graph of the five years from 1945 until 1950 is shown in Figure 4, and a graph of the five years from 1951 to 1955 is shown in Figure 5 . The activity of the nonHiroshima Japanese architects for the first five years immediately following the end of World War II, as shown in Figure 4, was 0\%. Local architects accounted for $91 \%$ of the building design work, so most of the reconstruction architecture in Hiroshima was in fact completed by local designers.

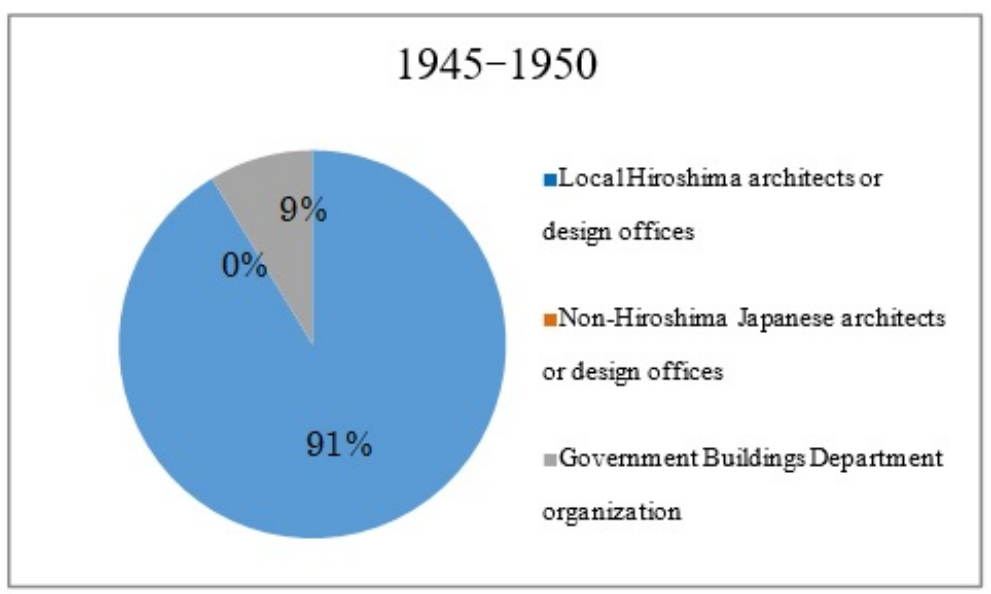

Figure 4. Architectural activity during 1945-1950 
As shown in Figure 5, at 29\%, there was a slight increase in the activity of non-Hiroshima Japanese architects from 1951; however, the activities of local architects accounted for $55 \%$ of the total construction activity.

The classification of the activities of the architects in Hiroshima immediately after the bombing, as clarified above, is summarized in Table 2. 1) The activity of local architects and design offices, including the Akatsuki Design Office, Isamu Ueno, the Yoshinari Kouchi architectural firm, the Murata \& Ohhata architectural firm, the Saburo Sugita design office, the Shirahani architect office, and the Toshio Kimura architectural design office, and the activities of the ARB architectural design firm can be confirmed. The activity of the Akatsuki Design Office was considerably high from 1945 to 1950. 2) As for the activity of the Prefecture City Buildings Department office, the Hiroshima Prefecture Buildings Department Division, the Hiroshima City Buildings Department Division, the Chugoku Shikoku Regional Construction Bureau Buildings Department Division, the Hiroshima City Waterworks Facilities Division, the Hiroshima Prefectural Housing Corporation, the Hiroshima Post Bureau Building Department, and the Chugoku Telecommunications Design Activities Department can be confirmed.

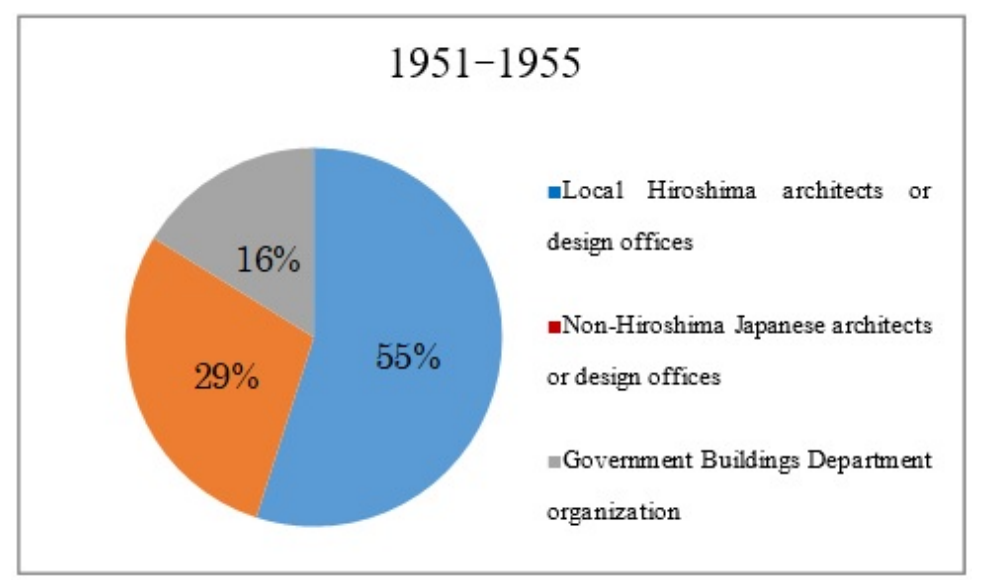

Figure 5. Architectural activity during 1951-1955

Note that the activity of the Hiroshima Local Buildings Department office immediately after the bombing was more noticeable than that before the war. Next, the activities of non-Hiroshima Japanese architects were classified into three categories based on factors, including architects/design offices, national maintenance company, general contractor, and nonHiroshima Japanese architects. 1) The activity of the architects and construction firms from the central district of Tokyo and Osaka, Kenzo Tange, Kunio Maekawa architectural Office, Ryotaro Tanaka, Togo Murano, Koji Nishimura architectural design Office, Toshio Yamashita architectural design Office, Kikuji Ishimoto, Masao Sasaguchi, Noboru Inaba, and Usaburo Kurokawa and the activities of Idota architectural design calculation office were confirmed. 2) As for the activity of the national maintenance and general contractors, the Supreme Court, Mizuno-gumi, Fujita-gumi, Kyoritsu-gumi, Mitsubishi Bank maintenance department, Fuji Bank maintenance department, Railway Administration Facilities Department Building Division, Asano-gumi, Toda-gumi, Soken Industry, Nippon Telegraph and Telephone Public Corporation Facilities Department Japan, and Takenaka Corporation and the design activities of other entities, such as Standard Oil Company, were confirmed. 3) The activity of the 
Hiroshima Branch Office of leading general contractors, Taisei Hiroshima Branch, and Shimizu Corporation Hiroshima branch and the design activities of Japan Denken Hiroshima Branch Office can be confirmed. 4) The activity of the non-Hiroshima Japanese architects and Isamu Noguchi and the design activities of ABCC Construction Department were confirmed.

Table 2. Activity types of architects and design offices in the reconstruction of Hiroshima immediately after World War II (1945-1955)

\begin{tabular}{|c|c|c|c|c|c|c|}
\hline \multirow[t]{2}{*}{ Period } & \multicolumn{3}{|c|}{$\begin{array}{l}\text { Non-Hiroshima Japanese architect } \\
\text { and design office }\end{array}$} & \multicolumn{2}{|c|}{$\begin{array}{l}\text { Local architect and design } \\
\text { office }\end{array}$} & \multirow[t]{2}{*}{ Remarks } \\
\hline & $\begin{array}{l}\text { Architect } \\
\text { and } \\
\text { design } \\
\text { office }\end{array}$ & $\begin{array}{l}\text { Design } \\
\text { organiza } \\
\text { tion }\end{array}$ & $\begin{array}{l}\text { Non- } \\
\text { Hiroshima } \\
\text { Japanese } \\
\text { architect }\end{array}$ & $\begin{array}{l}\text { Architect } \\
\text { and } \\
\text { design } \\
\text { office }\end{array}$ & $\begin{array}{l}\text { Buildings } \\
\text { department } \\
\text { organization and } \\
\text { general contractor } \\
\text { Hiroshima branch }\end{array}$ & \\
\hline 1945 & $\begin{array}{l}\bullet \text { Kenzo } \\
\text { Tange } \\
\text { (Commiss } \\
\text { ion) }\end{array}$ & & & & & \\
\hline 1946 & & & & $\begin{array}{l}\text { - Akatsuki } \\
\text { Design } \\
\text { Office }\end{array}$ & & \\
\hline 1947 & & & & $\begin{array}{l}\text { - Akatsuki } \\
\text { Design } \\
\text { Office }\end{array}$ & $\begin{array}{l}\text {-Hiroshima } \\
\text { Prefecture } \\
\text { Buildings } \\
\text { Department } \\
\text { Division }\end{array}$ & \\
\hline 1948 & & & & $\begin{array}{l}\text { - Akatsuki } \\
\text { Design } \\
\text { Office } \\
\text { - Saburo } \\
\text { Sugita } \\
\text { Design } \\
\text { Office }\end{array}$ & & $\begin{array}{l}\text { Peace } \\
\text { Memori } \\
\text { al } \\
\text { Cathedr } \\
\text { al } \\
\text { competit } \\
\text { ion }\end{array}$ \\
\hline 1949 & & & & $\begin{array}{l}\text { - Akatsuki } \\
\text { Design } \\
\text { Office } \\
\text { - Saburo } \\
\text { Sugita } \\
\text { Design } \\
\text { Office }\end{array}$ & & $\begin{array}{l}\text { Peace } \\
\text { Memori } \\
\text { al Park } \\
\text { Competi } \\
\text { tion }\end{array}$ \\
\hline 1950 & & & & $\begin{array}{l}\text { - Akatsuki } \\
\text { Design } \\
\text { Office } \\
\text { - Saburo } \\
\text { Sugita } \\
\text { Design } \\
\text { Office } \\
\text { - Shirahan } \\
\text { i Design } \\
\text { Office }\end{array}$ & $\begin{array}{l}\bullet \text { Chugoku } \\
\text { Shikoku Regional } \\
\text { Construction } \\
\text { Bureau Buildings } \\
\text { Department } \\
\text { Division }\end{array}$ & \\
\hline 1951 & & $\begin{array}{l}\bullet \text { Mitsub } \\
\text { ishi } \\
\text { Bank } \\
\text { Mainten } \\
\text { ance } \\
\text { Departm } \\
\text { ent\&Fuj } \\
\text { ita-gumi } \\
\bullet \text { Fujita- } \\
\text { gumi }\end{array}$ & $\begin{array}{l}\text { •Isamu } \\
\text { Noguchi }\end{array}$ & $\begin{array}{l}\text { - Akatsuki } \\
\text { Design } \\
\text { Office } \\
\text { - Saburo } \\
\text { Sugita } \\
\text { Design } \\
\text { Office } \\
\text { - Shirahan } \\
\text { i Design } \\
\text { Office }\end{array}$ & $\begin{array}{l}\text {-Hiroshima } \\
\text { Prefecture } \\
\text { Buildings } \\
\text { Department } \\
\text { Division } \\
\text {-Hiroshima City } \\
\text { Buildings } \\
\text { Department } \\
\text { Division } \\
\text { •Chugoku }\end{array}$ & \\
\hline
\end{tabular}




\begin{tabular}{|c|c|c|c|c|c|c|}
\hline \multirow[t]{2}{*}{ Period } & \multicolumn{3}{|c|}{$\begin{array}{l}\text { Non-Hiroshima Japanese architect } \\
\text { and design office }\end{array}$} & \multicolumn{2}{|c|}{$\begin{array}{l}\text { Local architect and design } \\
\text { office }\end{array}$} & \multirow[t]{2}{*}{ Remarks } \\
\hline & $\begin{array}{l}\text { Architect } \\
\text { and } \\
\text { design } \\
\text { office }\end{array}$ & $\begin{array}{l}\text { Design } \\
\text { organiza } \\
\text { tion }\end{array}$ & $\begin{array}{l}\text { Non- } \\
\text { Hiroshima } \\
\text { Japanese } \\
\text { architect }\end{array}$ & $\begin{array}{l}\text { Architect } \\
\text { and } \\
\text { design } \\
\text { office }\end{array}$ & $\begin{array}{l}\text { Buildings } \\
\text { department } \\
\text { organization and } \\
\text { general contractor } \\
\text { Hiroshima branch } \\
\text { Shikoku Regional } \\
\text { Construction } \\
\text { Bureau Buildings } \\
\text { Department } \\
\text { Division }\end{array}$ & \\
\hline 1952 & $\begin{array}{l}\bullet \text { Kenzo } \\
\text { Tange }\end{array}$ & $\begin{array}{l}\bullet \text { Mitsub } \\
\text { ishi } \\
\text { Bank } \\
\text { Mainten } \\
\text { ance } \\
\text { Departm } \\
\text { ent } \\
\text { \&Fujita- } \\
\text { gumi } \\
\text { • Fujita- } \\
\text { gumi } \\
\text { •Railwa } \\
\text { y } \\
\text { Adminis } \\
\text { tration }\end{array}$ & & $\begin{array}{l}\text { - Akatsuki } \\
\text { Design } \\
\text { Office } \\
\text { - Saburo } \\
\text { Sugita } \\
\text { Design } \\
\text { Office } \\
\text { - Shirahan } \\
\text { i Design } \\
\text { Office }\end{array}$ & $\begin{array}{l}\bullet \text { Hiroshima } \\
\text { Prefecture } \\
\text { Buildings } \\
\text { Department } \\
\text { Division } \\
\bullet \text { Hiroshima City } \\
\text { Buildings } \\
\text { Department } \\
\text { Division } \\
\bullet \text { Hiroshima City } \\
\text { Waterworks } \\
\text { Facilities }\end{array}$ & $\begin{array}{l}\text { Cenotap } \\
\mathrm{h} \text { for A- } \\
\text { bomb } \\
\text { victims }\end{array}$ \\
\hline 1952 & & $\begin{array}{l}\text { Facilitie } \\
\text { s } \\
\text { Departm } \\
\text { ent } \\
\text { Building } \\
\text { Division } \\
\text { - Asano- } \\
\text { gumi } \\
\text { - Toda- } \\
\text { gumi } \\
\text { • Souke } \\
\text { n } \\
\text { Industry }\end{array}$ & & & Division & \\
\hline 1953 & $\begin{array}{l}\bullet \text { Kenzo } \\
\text { Tange } \\
\text {-Kunio } \\
\text { Maekawa } \\
\text { - Ryotaro } \\
\text { Tanaka }\end{array}$ & $\begin{array}{l}\bullet \text { Taken } \\
\text { aka } \\
\text { Corpora } \\
\text { tion }\end{array}$ & $\begin{array}{l}\text { ABCC } \\
\text { Constructi } \\
\text { on } \\
\text { Departme } \\
\text { nt }\end{array}$ & $\begin{array}{l}\text { - Akatsuki } \\
\text { Design } \\
\text { Office } \\
\text { - Saburo } \\
\text { Sugita } \\
\text { Design } \\
\text { Office } \\
\text { - Isamu } \\
\text { Ueno } \\
\text { - Shirahan } \\
\text { i Design } \\
\text { Office }\end{array}$ & $\begin{array}{l}\text { •Hiroshima } \\
\text { Prefecture } \\
\text { Buildings } \\
\text { Department } \\
\text { Division } \\
\text { • Chugoku } \\
\text { Shikoku Regional } \\
\text { Construction } \\
\text { Bureau Buildings } \\
\text { Department } \\
\text { Division } \\
\text { • Japan Telephone } \\
\text { Public } \\
\text { Corporation } \\
\text { Facilities } \\
\text { Department } \\
\text { •Chugoku } \\
\text { Telecommunicati } \\
\text { ons Stations } \\
\text { Building } \\
\text { Department } \\
\text { •Hiroshima }\end{array}$ & \\
\hline
\end{tabular}




\begin{tabular}{|c|c|c|c|c|c|c|}
\hline \multirow[t]{2}{*}{ Period } & \multicolumn{3}{|c|}{$\begin{array}{l}\text { Non-Hiroshima Japanese architect } \\
\text { and design office }\end{array}$} & \multicolumn{2}{|c|}{$\begin{array}{l}\text { Local architect and design } \\
\text { office }\end{array}$} & \multirow[t]{2}{*}{ Remarks } \\
\hline & $\begin{array}{l}\text { Architect } \\
\text { and } \\
\text { design } \\
\text { office }\end{array}$ & $\begin{array}{l}\text { Design } \\
\text { organiza } \\
\text { tion }\end{array}$ & $\begin{array}{l}\text { Non- } \\
\text { Hiroshima } \\
\text { Japanese } \\
\text { architect }\end{array}$ & $\begin{array}{l}\text { Architect } \\
\text { and } \\
\text { design } \\
\text { office }\end{array}$ & $\begin{array}{l}\text { Buildings } \\
\text { department } \\
\text { organization and } \\
\text { general contractor } \\
\text { Hiroshima branch } \\
\text { Postal Service } \\
\text { Building } \\
\text { Department }\end{array}$ & \\
\hline \multirow[t]{19}{*}{1954} & •Murano & $\bullet$ Fujita- & & -Saburo & •Hiroshima & On \\
\hline & \& Mori & gumi & & Sugita & Prefecture & August \\
\hline & Design & -Shimiz & & Design & Buildings & 06 \\
\hline & Office & $\mathrm{u}$ & & Office & Department & 1949, \\
\hline & •Koji & Corpora & & - Isamu & Division & Hiroshi \\
\hline & Nishimura & tion & & Ueno & - Taisei & ma \\
\hline & Design & •Nikke & & - Yoshinar & Corporation & Peace \\
\hline & Office & $\mathrm{n}$ design & & i Kouchi & Hiroshima Branch & Memori \\
\hline & - Toshiro & -Obaya & & Design & $\bullet$ Hiroshima & al City \\
\hline & Yamashit & shi- & & Office & Postal Service & Constru \\
\hline & a Design & gumi & & •Onoki & Building & ction \\
\hline & Office & & & Constructi & Department & Law \\
\hline & -Kikuji & & & on & -Ministry of & (Law \\
\hline & Ishimoto & & & - ARB & Education & No. \\
\hline & -Masao & & & Design & Educational & 219) is \\
\hline & Sasaguchi & & & Office & Facilities & establish \\
\hline & & & & -Shirahan & Department & ed. \\
\hline & & & & i Design & Hiroshima & \\
\hline & & & & Office & $\begin{array}{l}\text { Construction } \\
\text { office }\end{array}$ & \\
\hline \multirow[t]{14}{*}{1955} & $\bullet$ Kenzo & •Fujita- & & - Yoshinar & •Hiroshima & Peace \\
\hline & Tange & gumi & & i Kouchi & Prefecture & Memori \\
\hline & & $\bullet$ Kyorit & & Design & Buildings & al is \\
\hline & & su-gumi & & Office & Department & complet \\
\hline & & & & - ARB & Division & ed \\
\hline & & & & Design & $\bullet$ Hiroshima City & Peace \\
\hline & & & & Office & Buildings & Memori \\
\hline & & & & -Shirahan & Department & \\
\hline & & & & i Design & Division & Museum \\
\hline & & & & Office & & \\
\hline & & & & -Murata\& & & complet \\
\hline & & & & Ohhata & & ed \\
\hline & & & & Design & & \\
\hline & & & & Office & & \\
\hline
\end{tabular}

In this way, in the actual activity of the architects in Hiroshima immediately after the bombing event, the local architects and design offices played a leading role. As summarized in Table 2, in the period immediately after the bombing event, Kenzo Tange conducted research sponsored by the commission of the Reconstruction Institute. Tange was active in two major competitions in Hiroshima. He was awarded the first prize in the Hiroshima Peace Memorial competition. The large role he played is known worldwide. Apart from his work, most other work corresponded to the activities of the design offices.

The major general contractors opened a branch office in Hiroshima in early 1955 when the Peace Center and the Peace Memorial Cathedral Hiroshima began to rise. Nevertheless, most building activity in the burned ruins immediately after the bombing event was mainly completed by local design offices. The vigorous activity of the local design offices was noteworthy. 
Table 3 lists the local design offices that were active in Hiroshima in the decade immediately after the war. From this list, the design activities of Hiroshima's first Akatsuki Design Office after the war have already been clarified in previous research. The Akatsuki Design Office was established as the first design office in Hiroshima after the atomic bombing event and became the model for the formation of many design offices that were constructed later. Its establishment had a great influence on the formation and development of its predecessor, the Hiroshima Architect Club in the Hiroshima Prefecture Architect Office Association. In the Akatsuki Design Office, architects Tadashi Murata, Yoshinari Kouchi, and Shoji Ohhata did the major design work.

Table 3. List of local architectural design offices in Hiroshima (1945-1955)

\begin{tabular}{lll}
\hline Year of establishment & Name of the office & Representative of the office \\
1946 & Akatsuki Design Office & Tadashi Murata \\
1948 & Saburo Sugita Design Office & Saburo Sugita \\
& Oda Design Office & Nousei Oda \\
& Wye Design Office & Yutaka Okamura \\
& ARB Design Office & Ryounoyichi Sasaki \\
& Katada Design Office & Tadao Katada \\
1949 & Kimura Design Office & Toshio Kimura \\
& Hasegawa Design Office & Unknown \\
1950 & Shirahani Design Office & Toshio Shibata \\
1951 & Yoshinari Kouchi Design Office & Yoshinari Kouchi \\
1952 & Araragi Design Office & Taro Yoshida \\
1953 & Nimura Design Office & Shiro Nimura \\
1954 & Murata \& Ohhata Design Office & Tadashi Murata \\
1955 & Kubo Design Office & Motoya Kubo \\
\hline
\end{tabular}

Note: This table is created on the basis of the documents and interviews provided by the Hiroshima Prefecture Architects Association.

These latter architects are the architects with the greatest influence in Hiroshima's rebuilding architecture.

There are many reconstructed buildings with which they are connected, and the heart blood of these three architects was poured into individual buildings. In particular, for the Children's Cultural Hall, the challenging design of a wooden building with a large span was created. The Agricultural Cooperative building was the first private post-war RC office building designed to harmonize with the surrounding environment. The Hiroshima Citizen's Hospital is made of 4-cm-thick precast concrete, which was created at the site to make thin eaves. Although these buildings did not attract the same attention as the Hiroshima Peace Park and the Hiroshima World Peace Shrine building, they are buildings that have an important place when discussing Hiroshima's reconstruction. Moreover, we find that they are interesting buildings in terms of their architecture.

In addition, Murata also played a role in communicating the plan of the construction of the World Peace Memorial Cathedral to director Nagashima by actively seeking the distribution of materials to the first private RC office in the country. As described above, the activities of the Akatsuki Design Office in the post-bomb Hiroshima reconstruction process did not entirely constitute the post-war building activity. They also clearly showed the activities of the architects themselves in Hiroshima's reconstruction process. In particular, it can be pointed out that the activities of local architects in Hiroshima dramatically increased. Other design offices and architects certainly played a role in Hiroshima's reconstruction process. As part of future research, we plan to analyze their contributions, thereby adding new 
content and an overview of the research on urban reconstruction through such work.

Thus, a certain understanding about the full scope of an architect or a design office involved in the reconstruction of Hiroshima is needed. Additionally, in the future, through a literature review and field survey, we plan to continue investigating the activities of individual architects and design offices. In particular, a full-scale investigation would focus on the architect Isamu Ueno, the Shirahani architect office, the Saburo Sugita design office, and Toshio Kimura Architects.

\section{THE ARCHITECTURAL ACTIVITIES AND FUNCTIONS OF THE AKATSUKI DESIGN OFFICE}

The Akatsuki Design Office was a team of $~ 10$ technicians, headed by Tadashi Murata, Yoshinari Kouchi, and Shoji Ohhata. The original office included president Tadashi Murata and members Youtaka Okamura, Minoru Shibata, Toshio Kimura, Yoshinari Kouchi, Akiyama (unknown), Toyotsuka Matsunaga, Kiyoto Oki, Kunihiko Fukuda, and two to three other architects. In a written statement, Yoshihiro Kawachi said, "In 1946, the only independent design office in Hiroshima was Akatsuki Design Office," emphasizing that Akatsuki Design Office was the first architectural design office in Hiroshima at that time (April 27, 1984, Chugoku Construction News Daily). As mentioned above, Akatsuki Design Office was the first architectural office established in Hiroshima after World War II, and it became the model for subsequent architectural design offices. First, the design office's stronghold was a corner of the Fukuya Department Store building damaged by the atomic bomb. Later, the office moved to the Hiroshima Agricultural Association building, which was designed by the office itself. Yoshinari Kouchi said, "The office space of the Akatsuki Design Office made use of the corridor of Fukuya department Store destroyed by the atomic bomb. It was partitioned by planks and had a space of less than $30 \mathrm{~m} 2$." Shoji Ohhata said, "Later, the Akatsuki Design Office was shifted from Fukuya department Store to a temporary housing at the construction site of the Hiroshima hospital, but because the typhoon blew the temporary housing, the design drawings of the office were all soaked through the rain." These events fully illustrate the difficulties of the architects' design activities in the post-war reconstruction period. At this time, to support the Akatsuki Design Office, Tetsuo Kuwata, the chairman of Hiroshima Agricultural Cooperative Credit Association, invited the employees of the Akatsuki Design Office to move to the Agricultural Cooperatives building.

From the Akatsuki Design Office, many architects launched independent design offices. After the independence of Kouchi Yoshinari in the year 1951, the Kouchi Yoshinari design office was set up. After the independence of Murata and Ohhata in 1954, the Murata \& Ohhata architecture design office was set up. Ohhata Syoji became independent in 1966 and established the Syoji Ohhata joint design office. Tadashi Murata became independent in 1966 and established the Murata mutual design office. From the Akatsuki Design Office to the Ohhata Syoji joint design office, the Murata mutual design office, and the Kouchi Yoshinari design office, three architectural design offices were set up. Shoji Ohhata said, 
"The Akatsuki Design Office did not disappear at that time but had a contemporaneous existence.” Thus, this one design office had a powerful design influence on all subsequent local Hiroshima architecture offices.

The process of the establishment and development of the Akatsuki Design Office is summarized in Table 4. Founded in 1946, the Akatsuki Design Office, with the atomic bomb explosion in the Hiroshima city as a background, carried out a vigorous building activity. Its first designs, the Asahi Newspaper Hiroshima branch building and Hiroshima Catholic Church Pastor Museum, were built at the end of 1946 in the vicinity of the tram road Komachi. These two buildings had wooden structures, and they represent the work of Tadashi Murata. Yoshinari Kouchi appraised the two works: "The buildings that could be seen in Hiroshima were the iron roof Barracks architecture or the trenches. In the center of Hiroshima Hondoori, only a few $3 \mathrm{~m} 2$ around the Stalls shop remained. However, there are two striking buildings at the Komachi-Tokyu tram road. There is the Asahi Newspaper Hiroshima branch building, $50 \mathrm{~m} 2$ of which corresponds to the Barracks architecture, but the use of burnt brick masonry in the fireplace chimney appears to be beautiful. Another building is the HataMachi Hiroshima Catholic Church Pastor Museum, which is built as a German wind wood structure building. These are both Murata's works, which appear to be considerably appealing; I was impressed." He described the horrors of the Hiroshima atomic bomb and praised the design of architect Murata.

Table 4. Chronology of the establishment and development of the Akatsuki Design Office 
Year Event

1946.4 Establishment of the Akatsuki Design Office; office location: fifth floor of the Fukuya Department Store building. The representative was Tadashi Murata.

1946.10 Yoshinari Kouchi joins the Akatsuki Design Office. The original Office president was Tadashi Murata. Members include Youtaka Okamura, Minoru Shibata, Toshio Kimura, Yoshinari Kouchi, Akiyama (unknown), Toyotsuka Matsunaga, Kiyoto Oki, Kunihiko Fukuda, and two to three other architects.

1948.6 The building plan of Yoshinari Kouchi won a prize in the design competition concerning the Hiroshima World Peace Memorial Hall.

1948.10 Architect Ohhata Syoji joined the Akatsuki Design Office. Akatsuki Design Office Shibata Minoru transferred to Fukuchiyama municipal government as the chief construction division.

1949.8 The building plan of Kouchi Yoshinari won a prize in the design competition concerning the Hiroshima Peace Center.

1950 Because of a fire incident, the Akatsuki Design Office in the Fukuya Department Store building was damaged, but the design drawings were not affected. In the same year, Kagawa Prefecture bought the Fukuya building and the Fukuya Department store opened. The Akatsuki Design Office relocated to temporary buildings at the public hospital building construction site, carrying out its business activities at this location.

1950.9 A typhoon caused the barracks office to collapse.

1951 Kuwada Tetuo, the chairman of the Hiroshima County Agricultural Credit Association, invited Akatsuki Design Office to move into the Agricultural Cooperative building.

1951.2 After the independence of Kouchi Yoshinari, the Kouchi Yoshinari architecture design office was established.

1954 After the independence of Murata and Ohhata, the Murata \& Ohhata Architecture Design Office was set up. Ohhata said that the Akatsuki Design Office did not disappear at that time and that there was a contemporary existence.

1961 Takada Teruhiro resigned from the Murata \& Ohhata Architecture Design Office and established the Takada Architecture Office.

1966 The Ohhata Syoji joint design office and the Murata mutual design office were established.

1966 Nshiki Akio resigned from the Kouchi Yoshinari Architecture Design Office and established the Institute of Urban Architecture.

Note: Table 4 refers to the information left behind in reports and other documents by late Kouchi Yoshinari and late Ohhata Syoji. It also includes information obtained by interviewing Ohhata Syoji.

By 1947, Yoshinari Kouchi basically chaired the design projects at the Akatsuki Design Office. The works designed by this office are the Hiroshima Jogakuin school building, Hiroshima Jogakuin auditorium, Sanyo Junior High School, and Koryo High School. To design these buildings, the architects worked almost every night until they were completely built. Their monthly salary was 500 yen (Kouchi, 1984). A cup of coffee and a box of tobacco were available for 10 yen each. After this time, Kouchi designed the Children's Cultural Hall (1948, Figure 6 (a)), the Hiroshima Gas Building (1949), and other projects. These buildings are all made of wood, but in Hiroshima, they also display the new characteristics of their design. The buildings that could be seen in Hiroshima at that time were the iron roof Barracks architecture or the trenches. In this context, the Hiroshima Gas Building was a brand-new building. Using wood and ceramic tiles, large restaurants and a report hall were set up. Some functions such as post-war conferences and entertainment were hosted here. The Children's Cultural Hall is a large-span wooden structure. At that time in Hiroshima, this building was considered unique. The goal of such a design was to bring hope to the contemporary and future post-war architectural design industry in Hiroshima. The following is a detailed analysis of the Children's Cultural Hall construction process and evaluation. 
The construction firm Shimizu was responsible for the construction of the Children's Cultural Hall, completed at a cost of 16,169,265 yen (Shimizu Corporation Hiroshima Branch, 1975). The building area is more than $1500 \mathrm{~m} 2$ and boasts a projection room, orchestra pit, and a 200m2 stage (Hiroshima Municipal Government, 1958). It also included a large wooden structure with 1000 spectator seats. Terunobu Fujimori hints, "The Japanese architectural world before the war was behind the world in terms of design and thought. In particular, the height and span are obviously lagging behind. For example, about height... Given it was such a big space, nobody thought it was a project of an architect. Overcoming these two backwardnesses is the great work of the Japanese architectural world after the war (Kashiwagi, Fujimori, et al. 1987). This statement raises the subject of post-war high-rise buildings, large spaces, and architects. The wooden, large-span Children's Cultural Hall built by Yoshinari Kouchi is not only important for the place it holds in Hiroshima's architectural history but also is an interesting building in the history of post-war Japanese large-space architecture. The hall is the only hall in the city with a projection booth, orchestra pit, and big stage. It is called "the children's paradise in the desert." Child-oriented gatherings, such as the Children's Culture Festival, were held here as were all kinds of cultural activities for adults. Therefore, it is also known as the "adult culture center" (Hiroshima Municipal Government, 1996). In addition to hosting cultural activities for children, the hall was used for theatrical performances, speeches, and assemblies. At that time, the famous Japanese comedians, Koreya Senda, Jukichi Uno, and Osamu Takizawa said, "It is surprising that a place such as this can have such a stage.” Howard Bell participated in a ceremony here and praised it saying that it offered "facilities that have not been seen in the United States.” The photos of the building of the Children's Cultural Hall were reported throughout the world and were publicized as a symbol of Hiroshima. At that time, Fumihiko Eto, a journalist from the Asahi Shimbun, praised the architects at Akatsuki Design Office saying that "the young architects had created frozen music" and had introduced a national vision (Asahi Newspaper Company, 1948). Kouchi said that at this time, to start constructing a building was to entrust a design office with design knowledge that was gradually recognized by society. The Children's Cultural Hall was dismantled in 1964. 


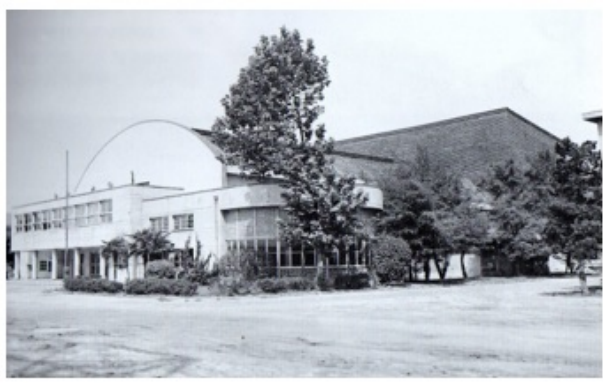

(a)

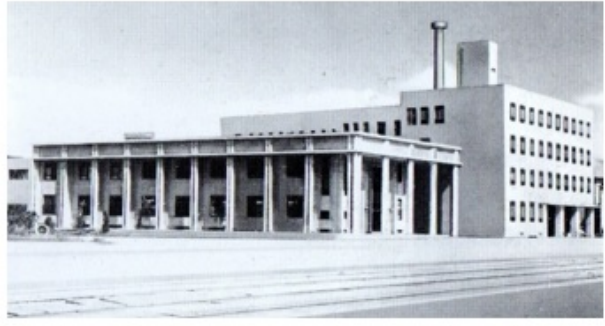

(c)

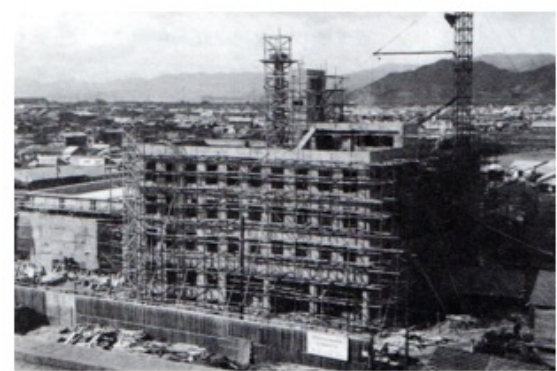

(b)

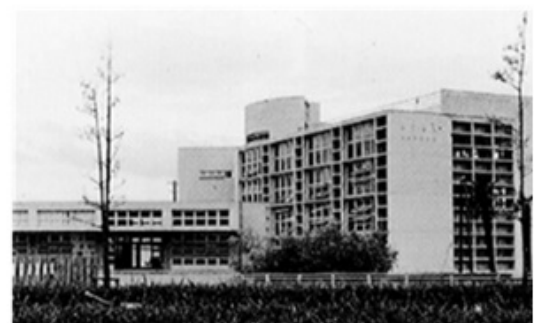

(d)

Figure 6. The architectural activities of Akatsuki Design Office. (a) Child Culture Hall (1948). (b) JA Building Construction scene (1949). (c) JA Building (1950). (d) Hiroshima City Hospital (1950).

In October 1948, architect Shoji Ohhata joined the Akatsuki Design Office. Subsequently, the main designs of the design firm were created by Tadashi Murata, Yoshinari Kouchi, and Shoji Ohhata. With the reconstruction process in Hiroshima, the construction of reinforced concrete structures is notable. Two such structures were designed in 1949 by the Akatsuki Design Office: Hiroshima Sinren and the JA Building (Figure 6 (b) and (c)). These two buildings were the first approved civil reinforced concrete structure office buildings in Japan. Tadashi Murata strove for results. These three architects together designed the Agricultural Cooperatives building, Taisho Marine \& Fire Insurance Hiroshima branch (1950), Social Insurance Hiroshima City Hospital (1951; Figure 6 (d)), the Hiroshima Department Store (1952), and other reinforced concrete structure buildings.

Started in 1949 and completed in 1950, the JA Building is a reinforced concrete structure with four levels (part of a two-floor office building). The construction cost was 68 million yen, and the construction firm was the Hazama Group. The construction process of this building is of profound significance in describing the revival of Hiroshima at that time.

Ohhata said that in 1947, the social status of the design firm was not high. The 1948 JA Building design required both steel and concrete to achieve a realistic design. After preparing cement, the Hazama Group obtained iron from the Wu City shipyard and transported it to Hiroshima, where it was converted into steel. This fully illustrates the status of the architects in the early rejuvenation and construction of Hiroshima and the difficulties of obtaining building materials. Shoji Ohhata said, "We designed the JA Building, I think, at a scale to be the country's largest building. In an age when cement and steel could not be obtained, it was able to be designed at such a scale and ignite the desire for design. Now, the building has become a complex building, but from a historical perspective, it is a symbol of Hiroshima's revival and construction and even a symbol of Japan's development after World War II (Hamaguchi and Ohhata, 1972). It can be 
said that the JA Building has made a great contribution to the revival of Hiroshima construction. The JA Building completed its 34-year historymaking mission; in 1984, it was demolished and reconstructed.

The Japanese building standards law was published on May 24, 1950, and a law specifying architectural training was published on May 02, 1950. At the same time, building materials began to sell freely and the architects began to be recognized by society.

In this period, the Akatsuki Design Office began to design the Social Insurance Hiroshima City Hospital. It was completed in 1951. The head of the first plan and the future comprehensive planning for the public hospital was Yoshinari Kouchi. Shimizu Construction Hiroshima branch was responsible for the construction. The Hiroshima City Hospital is built with reinforced concrete, the clinic is four floors (locally five layers), and the ward is two and three stories. According to the information provided by Shimizu Construction Hiroshima branch ((Shimizu Corporation Hiroshima Branch, 1975), the scale of the building is $3000 \mathrm{~m} 2$ and the construction cost was 49 million yen. The building shows graphic design planning, linear structure, clear functionality with a white wall, and a concrete shading board with a thickness of $4 \mathrm{~cm}$. Kouchi said, "The first phase of the form received the praise of the world-famous sculptor Sam Noguchi” (Kouchi, 1984). The first phase of the design of the Hiroshima City Hospital was a turning point. Kouchi, who resigned after five years with the early Akatsuki Design Office, founded the Kouchi Design Office. As Kouchi said, from then on, architects in Hiroshima began to be recognized and the design departments of the large Japanese design firms and construction companies began to enter Hiroshima's reconstruction.

Table 5. The main buildings designed by the Akatsuki Design Office in Hiroshima's postWorld War II revival and construction

\begin{tabular}{lll}
\hline Year of construction & Name of building & Structure \\
1946 & Parsonage & Wood structure \\
1946 & Asahi Newspaper Hiroshima Branch & Brick structure \\
1947 & Hiroshima Jogakuin School Building & Wood structure \\
1947 & Koryo High School & Wood structure \\
1948 & Sanyo Junior High School & Wood structure \\
1948 & Hiroshima Jogakuin Auditorium & Wood structure \\
1948 & Mainiti Newspaper Hiroshima Branch & Wood structure \\
1948 & Child Culture Hall & Wood structure \\
1949 & Hiroshima Gas Building & Wood structure \\
1950 & Agricultural Cooperative Building (JA & Reinforced concrete \\
& Building) & structure \\
1950 & Taisho Marine \& Fire Insurance Hiroshima & Reinforced concrete \\
& Branch & structure \\
1951 & Hiroshima City Hospital & Reinforced concrete \\
& & structure \\
1952 & Hiroshima Department Store & Reinforced concrete \\
& & structure \\
1952 & Hiroshima City Hospital Management & Reinforced concrete \\
& Building (extension) & structure \\
1953 & Second Ward and Nurse Building in & Reinforced concrete \\
1953 & Hiroshima City Hospital & structure \\
& Hiroshima Central Broadcasting Station & Reinforced concrete \\
1954 & (extension). & structure \\
& Hiroshima Central Broadcasting Bureau & Reinforced concrete \\
& Secretary of the Residential & structure \\
\hline
\end{tabular}

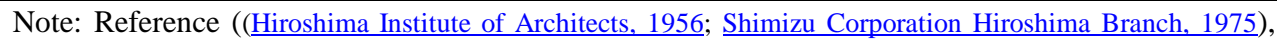
and confirmed by the interview with Murata, Kouchi and Ohhata. 
For the early period of Hiroshima's revival and construction, the main works of Akatsuki are summarized in Table 5. The design competition of the Akatsuki Design Office and its construction activities outside the Hiroshima area are summarized in Table 6.

Table 6. Design competition activities of the Akatsuki Design Office and their architectural activities outside the Hiroshima area

\begin{tabular}{ll}
\hline Year & Name of Building and Event \\
1948 & The Nagoya City Hospital was designed. \\
1948 & The Ube Hospital was designed. \\
1948 & For the design competition of the Hiroshima Peace Memorial Church, Li \\
& Ming won the quasi-masterpiece award. \\
1949 & The Tokuyama City Hospital was designed. \\
1949 & $\begin{array}{l}\text { For the design competition of the Hiroshima Peace Memorial Park, Li } \\
\text { Ming won the masterpiece award. }\end{array}$ \\
& $\begin{array}{l}\text { Hideshima (Waseda University), executive director of the Japan Urban } \\
\text { Planning Society, visited Hiroshima. The Akatsuki Design Office invited } \\
\text { to design the Paris-type urban renewal plan, and Kouchi designed the } \\
\text { bird's eye view, but this plan was not implemented. } \\
\text { Attending the design competition of the Hiroshima Peace Memorial } \\
\text { Bridge. }\end{array}$ \\
\hline
\end{tabular}

Note: Reference (Kouchi, 1984), and confirmed by interviews with Li Ming.

As mentioned above, the revival building by the Akatsuki Design Office dominated the efforts of the time. The blood of its three main architects was poured into each building. Kouchi said, "The early design office at that time designed $70 \%$ of the main buildings in the city of Hiroshima" (Kouchi, 1983). Tadashi Murata said, "The buildings we built during the Renaissance were dismantled year by year. However, when it comes to Hiroshima, I cannot keep silent about the blood, the sweat, and the tears of the people who love Hiroshima” (Murata, 1983). During the post-war reconstruction of Hiroshima, many builders and technicians devoted their time to it. In addition, as Kouchi reviewed, "With the revival of Hiroshima after the atomic bombing event, the bridges, roads, drainage, gas, electricity, and other infrastructure facilities were the foundation for the spirit of the people who survived the difficulties associated with food and housing with enthusiasm. However, what can be seen is the work of the designers and carpenters and the achievements of the gradually established construction workers.” The role of local architecture design firms in Hiroshima's revival and construction was pivotal.

\section{CONCLUSIONS}

We attempted to discuss the architects and the actual activities associated with the reconstruction of Hiroshima.

1) Although many buildings in the reconstruction process were constructed by others, a major role was played by local design offices. In particular, the major contributions of the local design offices, including the Akatsuki Design Office, were observed.

2) On August 06, 1949, the Hiroshima Peace Memorial City Construction Law was promulgated and two major competitions - the Peace Memorial Cathedral competition and the Hiroshima Peace Center competition-were conducted. Many architects and future architects from all over the country participated in these competitions with enthusiasm. They exhibited the stunning Japanese-style architecture. These competitions 
not only played a significant role in promoting the full-scale reconstruction of Hiroshima but also played a major role in the development of post-war Japanese architecture. Tange was active in the two major competitions in Hiroshima and won the Hiroshima Peace Memorial competition. He is known around the world for the large role he has played.

3) From the early-mid 1950s, when the Hiroshima Peace Center and the Peace Memorial Cathedral began to be built, we can observe that a nonHiroshima Japanese architect or the design office and leading general contractors began to seriously enter the architectural arena in Hiroshima.

4) Overall, we can observe that most of the building activity that occurred in the burned ruins immediately after the bombing event was performed by local design offices. The vigorous activity of the local design offices deserves attention and should not be overlooked as a subject of research to better understand the reconstruction of the local city. Because this study focuses on the elucidation of the actual activities performed by the architects, it was impossible to address the specific reconstruction activities of individual architects. We would like to continue the research in this regard in the future.

\section{ACKNOWLEDGMENTS}

This study was supported by a grant from the JSPS Scientific Research (C) (Research Project Number: 26420656).

\section{PHOTO SOURCE}

Figure 1 (a)-(k) and Figure 6 (d): Hiroshima Institute of Architects. (1956). Architecture of Hiroshima (1945-1955). Hiroshima, Japan: Chugoku photo printing press.

Figure 6 (a): Hiroshima Municipal Government (1996.3.31). The History of Hiroshima, the 50 Years of Community and Life After the War. Hiroshima, Japan: Hiroshima City Hall.

Figure 6 (b)-(c): 30 years of review: Yomuseyo. (1983). Hiroshima, Japan: Hiroshima building administrative Association.

\section{REFERENCES}

Asahi Newspaper Company. (1948). Asahi Shimbun, 1948.5.4, Tokyo.

Fujimori, T. (1982). Meiji Tokyo Project, Iwamasa Shoden, Tokyo.

Fukushima University International Disaster Revival Research Team. (2014). International Comparison of Recovery from the Great East Japan Earthquake, Hasskusya, Tokyo.

Hamaguchi, R., \& Ohhata, S. (1972). The Architect's Personality and Works: The Pursuit of People and Buildings, Ohhata Joint Architecture Design Office, Hiroshima.

Hiroshima Institute of Architects. (1956). Architecture of Hiroshima (1945-1955), Chugoku photo printing press, Hiroshima.

Hiroshima Institute of Architects. (1962). Architecture of Hiroshima (1956-1961), Chugoku photo printing press, Hiroshima.

Hiroshima Institute of Architects. (1983). Architecture of Hiroshima (1962-1982), Chugoku photo printing press, Hiroshima.

Hiroshima Municipal Government. (1958). The History of the New Hiroshima City, Fourth Volume, the History of Culture and Customs, Hiroshima City Hall, Hiroshima.

Hiroshima Municipal Government. (1985). Hiroshima atomic bomb 40 years 
history/reconstruction of the city, Hiroshima City Hall, Hiroshima.

Hiroshima Municipal Government. (1996). The History of Hiroshima: the 50 Years of Community and Life After the War, Hiroshima City Hall, Hiroshima.

Ishimaru, N. (1990). Transfiguration of urban form and urban landscape-a century that woke Hiroshima, Hiroshima City Archives Bulletin No.13, Hiroshima.

Ishimaru, N., Li, M., \& Okagawa, M. (2002). “A Life and Works Of Architect Shoji Ohhata: A study on the activities of the architect in the Hiroshima district”, J.Archit. Plann. Environ. Eng., AIJ, (557), 339-345. https://doi.org/10.3130/aija.69.191_1

Japanese Ministry of Construction. (1928). The reconstruction of Tokyo, Tokyo Municipal Council, Tokyo.

Japanese Ministry of Construction. (1959). The first volume: The disaster revival plan, career series, Tokyo Municipal Council, Tokyo.

Kaji, H., Izumi, J., \& Yamamoto, Y. (2012). Suggestion to the East Japan Great Earthquake Disaster Restoration and Reconstruction, Gihodo Shuppan, Tokyo.

Kashiwagi, H., Fujimori, T., Funo, S., \& Matsuyama, I. (1987). Architectural Writer Era, Riff Furnace Port, Inc., Tokyo.

Koshizawa, A., \& Goto, X. (2011). Great earthquake disaster and Tokyo revival. Tokyo, Japan: Chikuma Shobo.

Kouchi, Y. (1983). 30 years of review: Yomuseyo, Hiroshima Building Administrative Association, Hiroshima, Japan.

Kouchi, Y. (1984). Architecture of Hiroshima, Chugoku Construction Newspaper, Hiroshima.

Li, M. (2012). Hiroshima of reconstruction and Architects, Miyaobi press, Kyoto.

Li, M., \& Ishimaru, N. (2006). "The Study on The Activities And Their Features Of Architects In Hiroshima Before World War Two: The Study On The Architects Activity Form And Feature In Local City”, Journal of Architecture and Planning (Transactions of AIJ), 71(608), 197-204. https://doi.org/10.3130/aija.71.197_4

Li, M., \& Ishimaru, N. (2007). “Architect Yoshinari Kouchi's History and His Works”, Journal of Architecture and Planning (Transactions of AIJ), 72(617), 215-222. https://doi.org/10.3130/aija.72.215_3

Li, M., \& Ishimaru, N. (2014). “Architect Benji Toyoda' S History and His Works”, Journal of Architecture and Planning (Transactions of AIJ), 79(703), 2077-2084. https://doi.org/10.3130/aija.79.2077

Murata, T. (1983). Ushered in the greeting of the 30th anniversary: President of the Hiroshima Municipal Construction Administration Association, Hiroshima Building Administrative Association, Hiroshima.

Shimizu Corporation Hiroshima Branch. (1975). The foundation of 30 years, Hiroshima.

Tanaka, T. (2006). Reconstruction and Living Space in Tokyo, The University of Tokyo press, Tokyo.

Tokyo Municipal Government. (1932). Regional planning and consolidation of Tokyo reconstruction (the first), Kawaguchi Printing Institute, Tokyo.

Yamamoto, H. (2014). Studies of Disaster Response: Design of Cultural Reconstruction, Kyoto University academic publications, Kyoto. 